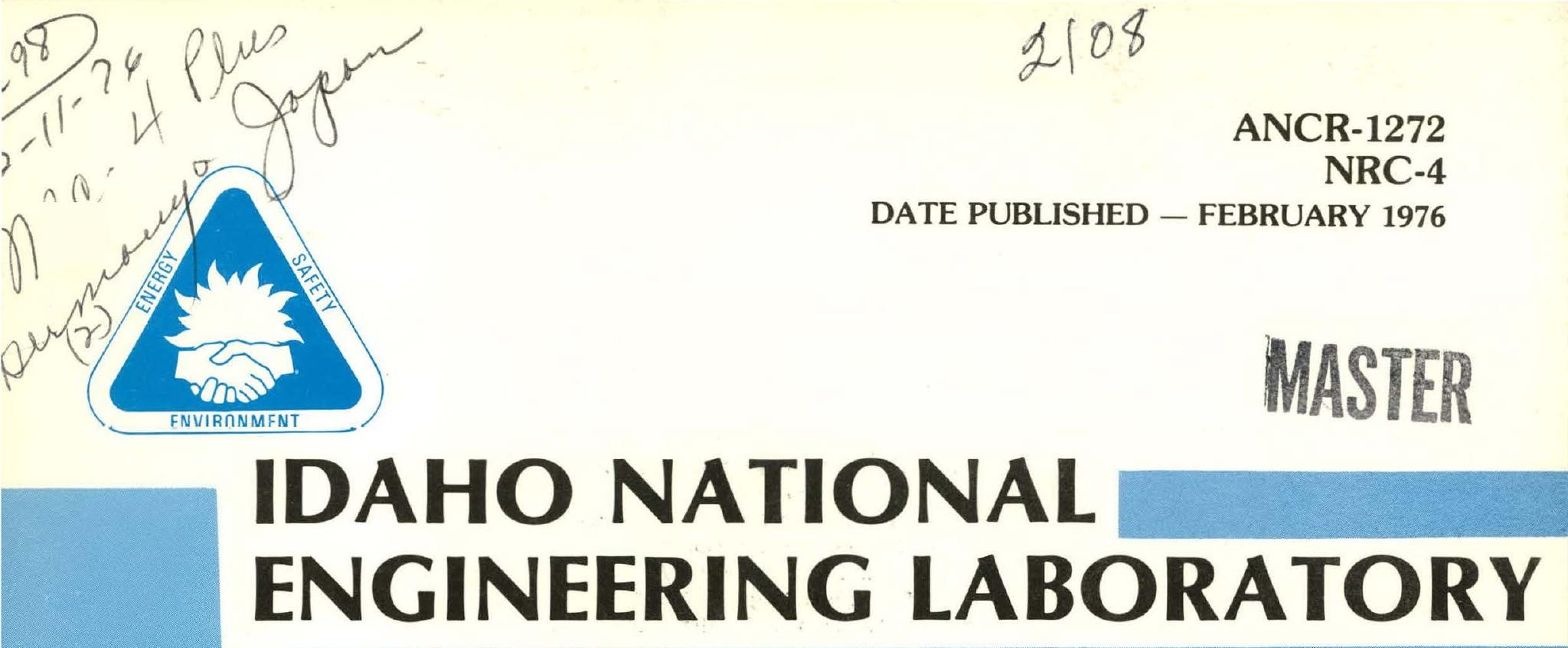

CHARACTERISTICS, STABILITY, AND SHORT-WAVELENGTH PHENOMENA IN TWO-PHASE EQUATION SYSTEMS 


\section{DISCLAIMER}

This report was prepared as an account of work sponsored by an agency of the United States Government. Neither the United States Government nor any agency Thereof, nor any of their employees, makes any warranty, express or implied, or assumes any legal liability or responsibility for the accuracy, completeness, or usefulness of any information, apparatus, product, or process disclosed, or represents that its use would not infringe privately owned rights. Reference herein to any specific commercial product, process, or service by trade name, trademark, manufacturer, or otherwise does not necessarily constitute or imply its endorsement, recommendation, or favoring by the United States Government or any agency thereof. The views and opinions of authors expressed herein do not necessarily state or reflect those of the United States Government or any agency thereof. 


\section{DISCLAIMER}

Portions of this document may be illegible in electronic image products. Images are produced from the best available original document. 


\section{Printed in the United States of America \\ Available from}

National Technical Information Service

U. S. Department of Commerce

5285 Port Royal Road

Springfield, Virginia 22161

Price: Printed Copy $\$ 5.00$; Microfiche $\$ 2.25$
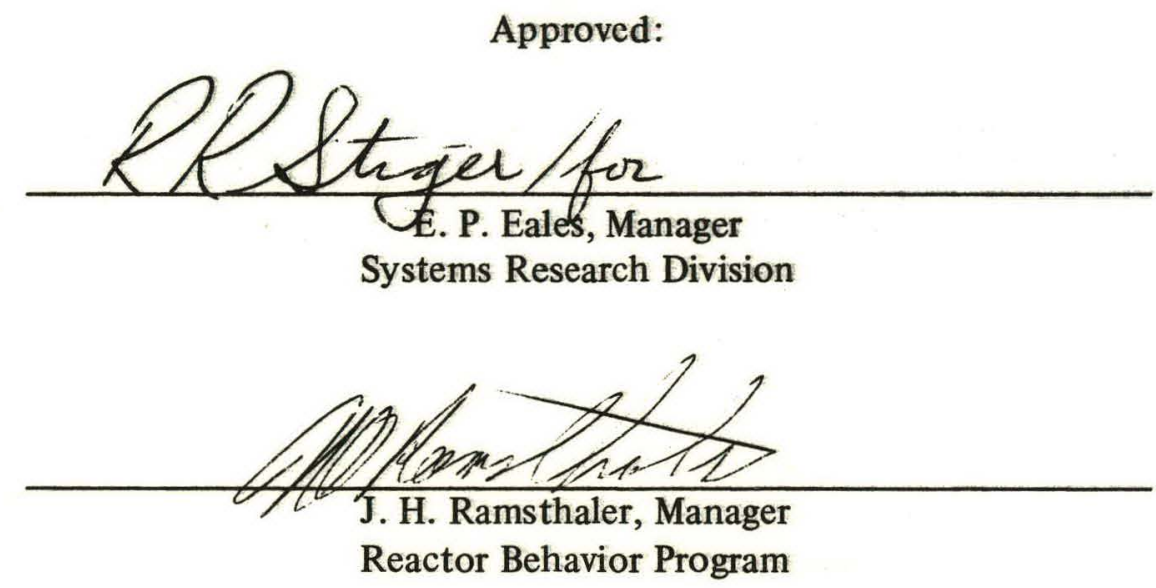

NOTICE

This report was prepared as an account of work sponsored by the United States Government. Neither the United States nor the Energy Research and Development Administration, nor the Nuclear Regulatory Commission, nor any of their employees, nor any of their contractors, subcontractors, or their employees, makes any warranty, express or implied, or assumes any legal liability or responsibility for the accuracy, completeness or usefulness of any information, apparatus, product or process disclosed, or represents that its use would not infringe privately owned rights. 


\title{
CHARACTERISTICS, STABILITY, AND SHORT-WAVELENGTH PHENOMENA IN TWO-PHASE FLOW EQUATION SYSTEMS
}

\author{
by
}

J. D. Ramshaw

J. A. Trapp

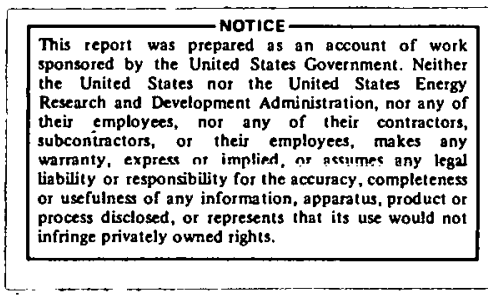

AEROJET NUCLEAR COMPANY

Date Published - February 1976

PREPARED FOR THE

U.S. ENERGY RESEARCH AND DEVELOPMENT ADMINISTRATION

IDAHO OPERATIONS OFFICE

UNDER CONTRACT NO. E(10-1)-1375 


\begin{abstract}
- The occurrence and significance of complex characteristics in two-phase flow equation systems are clarified by a detailed analysis of separated two-phase flow between two parallel plates. The basic.system of one-dimensional, two-phase flow equations for this problem possesses complex characteristics, exhibits unbounded instabilities in the short-wavelength limit, and constitutes an improperly posed initial value problem. These difficulties have led some workers to propose major modifications to the basic equation system. The relatively minor modification of introducing surface tension is shown to be sufficient to render the characteristics real, to stabilize short-wavelength disturbances, and to produce a properly posed problem. For a given value of the surface tension, the basic equation system thus modified is shown to predict correctly the evolution of small-amplitude disturbances having wavelengths long compared to the plate spacing. A formula is given for the artiticial surface tension necessary to stabilize wavelengths of the order of the mesh spacing in a finite-difference numerical calculation. A brief discussion is given concerning the expected behavior of surface tension as compared to viscosity in the nonlinear regime. The general relationship between characteristics and stability is discussed in Appendix A.
\end{abstract}


CONTENTS

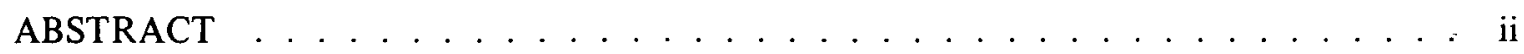

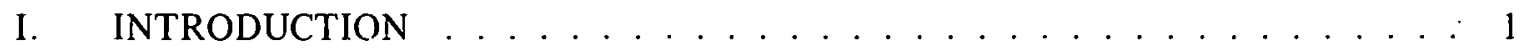

II. ADDITION OF SURFACE TENSION TO THE BASIC EQUATION SYSTEM . . 4

III. LINEAR STABILITY ANALYSIS OF THE SIGMA EQUATION SYSTEM . . . . 7

IV. EXACT TWO-DIMENSIONAL STABILITY ANALYSIS $\ldots \ldots \ldots \ldots$

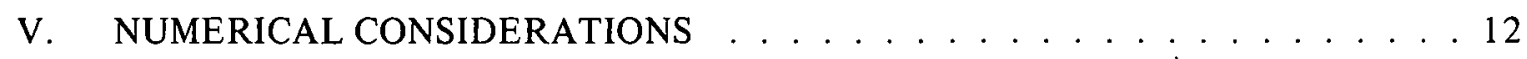

VI. CONCLUDING REMARKS $\ldots \ldots \ldots \ldots \ldots \ldots$

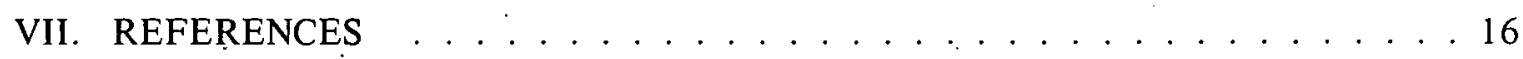

APPENDIX A - RELATIONSHIP BETWEEN CHARACTERISTICS

AND STABILITY $\ldots \ldots \ldots \ldots$

\section{FIGURE}

1. Geometry of separated two-phase flow between two parallel plates $\ldots \ldots \ldots 1$ 


\section{CHARACTERISTICS, STABILITY, AND SHORT-W $\Lambda$ VELENGTH PHENOMENA IN TWO-PHASE FLOW EQUATION SYSTEMS}

\section{INTRODUCTION}

Recently, much confusion has arisen in connection with the problem of complex characteristics in two-phase flow equation systems [1]. The problem may be illustrated by considering the case of separated two-phase flow between two parallel plates in zero gravity, as represented in Figure 1. For simplicity, the fluids are assumed inviscid and barotropic, with no mass exchange between them. The most straightforward way to describe this situation would be to apply to each phase the well-established one-dimensional equations for flow in a variable. area duct ${ }^{[2]}$. This approach leads at once to the fulluwing system of two-phase flow equations:

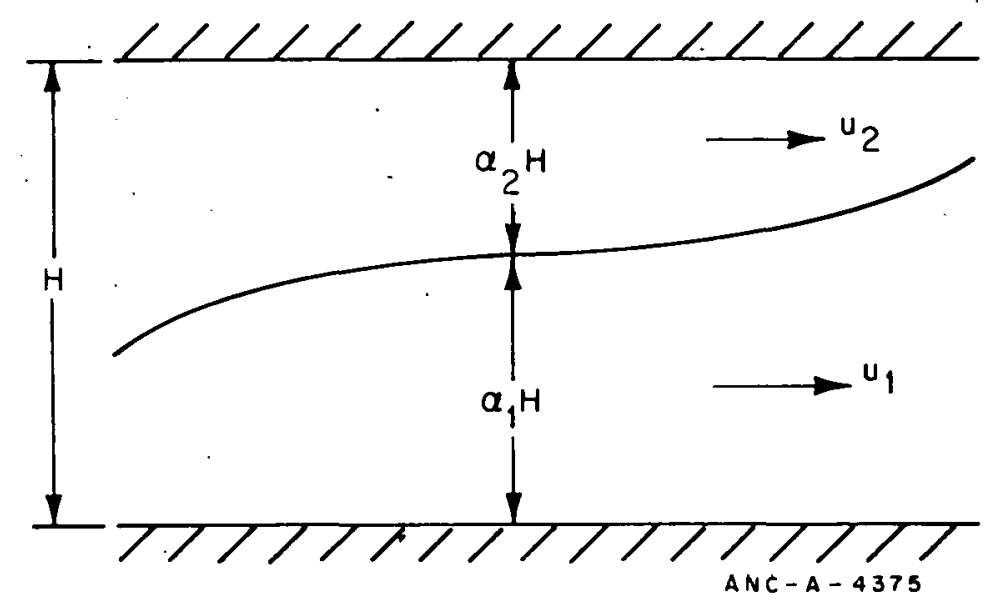

Fig. 1 Geometry of separated two-phase flow between two parallel plates.

$$
\begin{gathered}
\frac{\partial}{\partial t}\left(\alpha_{i} \rho_{i}\right)+\frac{\partial}{\partial x}\left(\alpha_{i} \rho_{i} u_{i}\right)=0 \quad(i=1,2) \\
\frac{\partial}{\partial t}\left(\alpha_{i} \rho_{i} u_{i}\right)+\frac{\partial}{\partial x}\left(\alpha_{i} \rho_{i} u_{i}^{2}\right)+\alpha_{i} \frac{\partial p}{\partial x}=0 \quad(i=1,2) \\
\alpha_{1}+\alpha_{2}=1
\end{gathered}
$$

where $\alpha_{i}, \rho_{i}$, and $u_{i}$ are, respectively, the volume fraction, density, and velocity of phase $\mathrm{i}$, and $\mathrm{p}$ is the pressure; all of these quanitites are functions of the distance $\mathrm{x}$ and the time $\mathrm{t}$. Equations (1) to (3) will be referred to as the basic equation system. 
Equations (1) to (3) are now widely recognized to possess complex characteristics ${ }^{[1]}$. (The term "characteristics" is used here in accord with standard mathematical terminology $[3,4]$ and is explained in Appendix A.) Consequently, the solutions are unstable at very short wavelengths, and the equations constitute an improperly posed initial-value problem (further discussion is given in Appendix A). However, opınions differ as to the correct interpretation of these facts and the proper course of action [1].

Some workers have intimated that complex characteristics are necessary to the description of physical instabilities [1]. This beliel is shown to be crroneout; il appareintly stems from confusion about the relation between characteristics and stability. This relation is discussed in Appendix A, where characteristics and stability are, in general, shown to be related only in the limit of large frequency and short wavelength.

One might instead adopt the related, but less extreme, position that equations having complex characteristics may correctly describe physical instabilities. This view, when properly qualified, is correct; but the qualification is a rather serious one: the wavelengths involved must be sufficiently long. Equations having complex characteristics can indeed provide a correct description of long-wavelength physical instabilities; Equations (1) to (3) themselves are of this type. However, in all such cases, the results will become increasingly erromeroms as the wavclength is decreased. Eventually, at short enough wavelengths, cquations having complcx characteristics will predict. unphysical inștahilities.

Probably the majority of investigators believe that in two-phase flow, as in other areas of physics and engineering science, physical phenomena should find their mathematical expression in terms of properly posed problems [4,5]. Then Equations (1) to (3) must be incomplete or incorrect in some essential physical respect, and one may expect that when their physical deficiencies arc corrected, their characteristics will become real. The authors of this report share this view, but differ from some of its other adherents in the type of recommended modifications to Equations (1) to (3). This report shows that the complex characleristics and their attendant difficulties can be removed simply by including known physical effects which, while for the most part negligible, become important at short wavelengths (or high frequencies, or both). The inclusion of such effects does not alter the behavior of the equation system at long wavelengths. This point is important because, as will bc seen, Equations (1) to (3) as they stand predict correct long-wavelength behavior for the case of small perturbations about a uniform steady state solution. Other authors, disregarding this fact, have proposed modifications to Equations (1) to (3) which alter their behavior at both short and long wavelengths. The most common examples of such modifications are (a) modifying the pressure gradient term in Equation (2) to read $\partial\left(\alpha_{i} p\right) / \partial x^{[1,6]}$, and (b) addition of first-order differential terms, nominally representing various interface forces, to the right-hand side of Equation (2) ${ }^{[7]}$.

The particular short-wavelength physical effect considered in this report is that of surface tension. This effect was chosen primarily for reasons of mathematical simplicity. Other short-wavelength effects, viscosity in particular, give rise to similar behavior but are much more difficult to treat rigorously in connection with the exact two-dimensional analy sis of Section IV. 
When surfacc tension is introduced into Equations (1) to (3), the characteristics are rendered real, short-wavelength disturbances are stabilized, and the initial-value problem becomes properly posed. Since the effect of surface tension is confined to short wavelengths, the resulting equation system predicts that long wavelengths remain unstable, and it allows such instabilities to be calculated. This long-wavelength instability is physical in nature; it is simply the familiar classical Helmholtz instability, occurring in a hnunded region. In fact, much of the present motivation and development exploits this correspondence. The conventional Helmholtz problem is known to be ill posed in the absence of surface tension and well posed when surface tension is included ${ }^{[8]}$, which in the one-dimensional, two-phase description corresponds to complex and real characteristics, respectively. In addition, the exact solution of the Helmholtz problem between two parallel plates including surface tension can readily be obtained by standard methods and used for comparison with the results of the one-dimensional, two-phase equations.

The remainder of the report is organized as follows. In Section II, the basic equation system is generalized to allow for surface tension, and the characteristics are then shown to become real. In Section III, a linear stability analysis of the equation system including surface tension is performed. If the surface tension $\sigma$ is zero, then all wavelengths are found to be unstable, and the problem is ill posed; whereas if $\sigma>0$, short wavelengths are stable, and the problem is well posed. In Section IV, the problem of separated two-phase flow is considered as a classical Helmholtz instability occurring in a bounded region. The results of an exact two-dimensional stability analysis, including the effect of surface tension, are summarized and compared with the results of Section III. The results of Sections III and IV are in agreement, for an arbitrary value of $\sigma(\sigma \geqslant 0)$, provided that the wavelength is long compared to the plate spacing. A brief discussion of numerical considerations is given in Section V. Section VI contains a few concluding remarks including comments about the expected behavior of surface tension as compared to viscosity in the nonlinear regime. Appendix A discusses the connection between characteristics and stability for a general system of first-order, quasi-linear partial differential equations in the independent variables $x$ and $t$. 


\section{ADDITION OF SURFACE TENSION TO THE BASIC EQUATION SYSTEM}

The effert of surfare tensinn can easily he incorporated into Equations (1) to (3) by regarding the two phases as having slightly different pressures, the two pressures being coupled by the usual surface tension interface condition. Thus:

$$
\begin{gathered}
\frac{\partial}{\partial t}\left(\alpha_{i} \rho_{1}\right)+\frac{\partial}{\partial x}\left(\alpha_{i} \rho_{i} u_{i}\right)=0 \quad(i=1,2) \\
\frac{\partial}{\partial t}\left(\alpha_{i} \rho_{i} u_{1}\right)+\frac{\partial}{\partial x}\left(\alpha_{i} \rho_{i} u_{i}^{2}\right)+\alpha_{i} \frac{\partial p_{i}}{\partial x}=0 \quad(i=1,2) \\
\alpha_{1}+\alpha_{2}=1 \\
p_{1}-p_{2}=\frac{\sigma}{R}
\end{gathered}
$$

whcre $p_{i}$ is the pressure of phase $i, \sigma$ is the surface tension, and $R$ is the radius of curvature of the interface, given by

$$
\frac{1}{R}=-H \frac{\partial^{2} \alpha}{\partial x^{2}}\left[1+H^{2}\left(\frac{\partial \alpha}{\partial x}\right)^{2}\right]^{-3 / 2}
$$

and where $\mathrm{H}$ is the plate spacing.

The assumption is made that $\mathrm{H}^{2}\left(\partial \alpha_{1} / \partial \mathrm{x}\right)^{2} \ll 1$ since if such is not the case, the one-dimensional, variable-area-duct equations on which Equations (1) to (3) were based become unrealistic anyway: By combining Equations (7) and (8) under this assumption, the . following is obtalned:

$$
p_{1}-p_{2}=-\sigma 11 \frac{\partial^{2} \alpha_{1}}{\partial x^{2}}
$$

Equations (4), (5), (6), and (9) are referred to as the sigma equation system.

In order to determine the characteristics of the sigma equation system, the system must be converted to first-order form. This conversion can be done by means of the substitution $\beta=\partial \alpha_{1} / \partial x$, so that Equation (9) is replaced by the equations

$$
\begin{gathered}
p_{1}-p_{2}+\sigma H \frac{\partial \beta}{\partial x}=0 \\
\frac{\partial \alpha_{1}}{\partial x}-\beta=0 .
\end{gathered}
$$


The two phases are assumed barotropic, so

$$
\rho_{i}=f_{i}\left(p_{i}\right) \quad(i=1,2)
$$

where the functions $f_{i}$ may be considered known. The algebraic Relations (6) and (12) may be used to eliminate $\alpha_{2}, \rho_{1}$, and $\rho_{2}$ as dependent variables. When these variables have been eliminated, the equation system may be written in matrix form as follows:

$$
A \cdot \frac{\partial v}{\partial t}+B \cdot \frac{\partial v}{\partial x}+\stackrel{c}{\sim}=0
$$

where $\mathrm{v}$ is a column vector of dependent variables:

$$
\underset{\sim}{\mathrm{v}}=\left(\mathrm{p}_{1}, \mathrm{p}_{2}, \mathrm{u}_{1}, \mathrm{u}_{2}, \alpha_{1}, \beta\right)^{\mathrm{T}}
$$

(the superscript $\mathrm{T}$ denoting the matrix transpose), $\mathbf{A}$ and $\mathbf{B}$ are matrices:

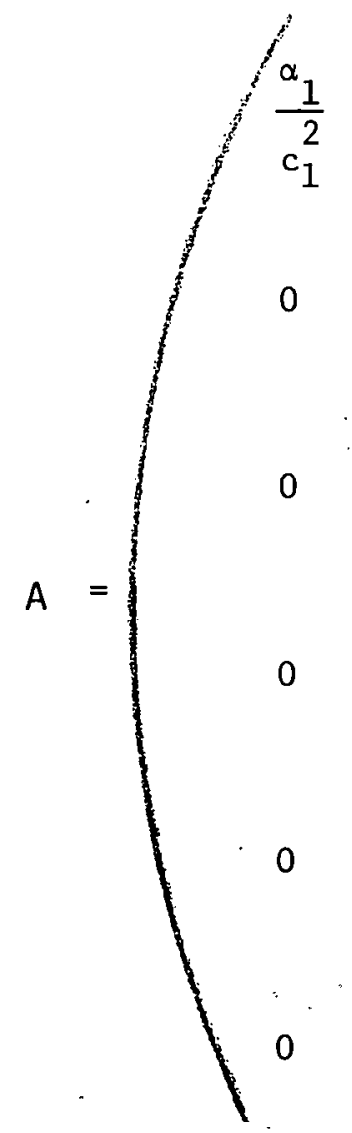

0

0

0

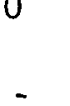$$
\frac{\alpha_{2}}{c_{2}^{2}}
$$

0

0<smiles>[2H][10BH2]</smiles>

0

$$
\alpha_{1} \rho_{1}
$$

0

0

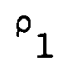

2 

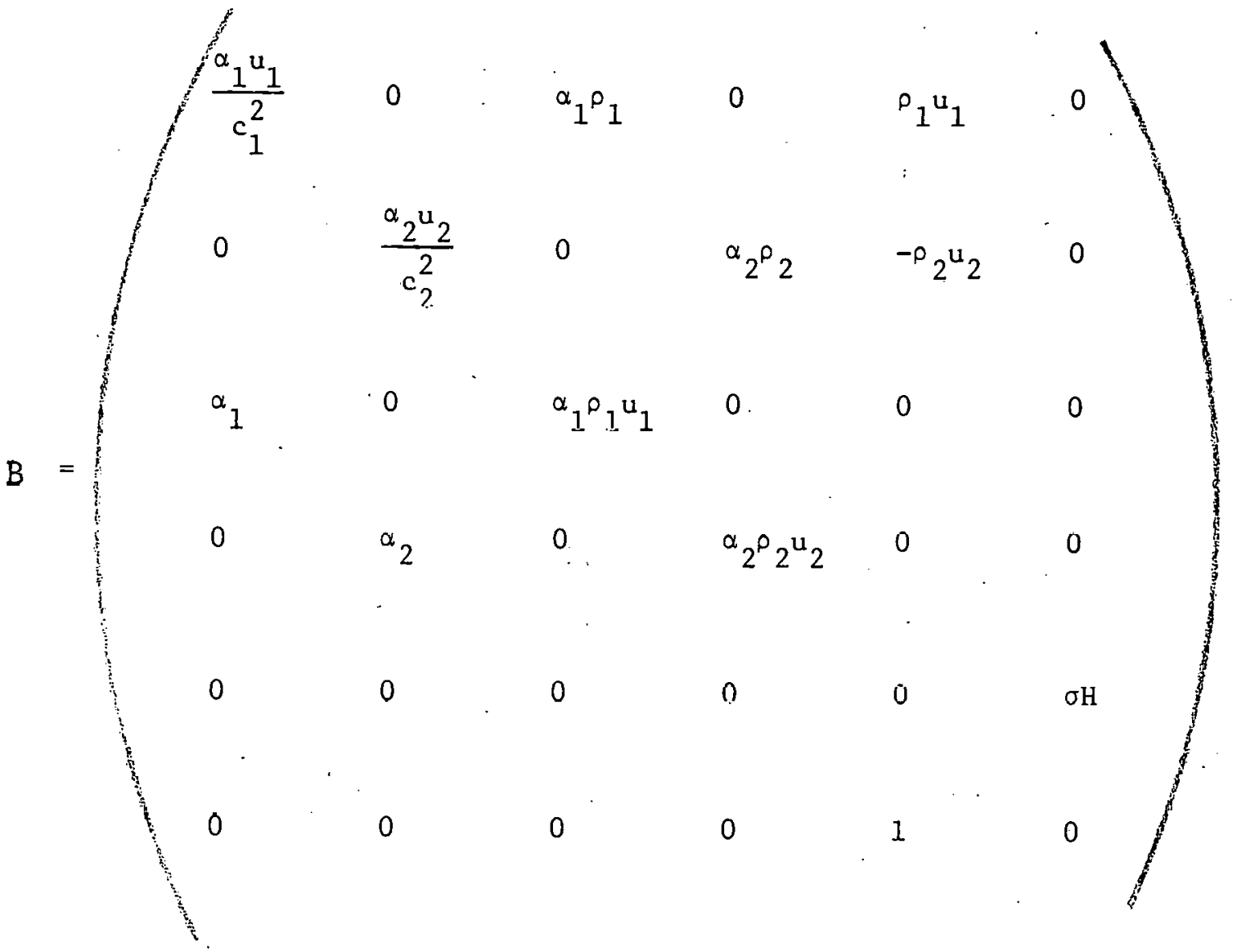

in which $c_{\mathrm{i}}$ denotes the sound speed $\left(\mathrm{dp} \mathrm{p}_{\mathrm{i}} / \mathrm{d} \rho_{\mathrm{i}}\right)^{1 / 2}$ of phase $\mathrm{i}$, to be evaluated from Equation (12), and $\underset{\sim}{c}$ is a "source" vector:

$$
\subseteq=\left(0,0,0,0, \mathrm{p}_{1}-\mathrm{p}_{2},-\beta\right)^{\mathrm{T}} \text {. }
$$

The characteristics $\mu$ are defined hy the equation

$$
\operatorname{det}(A-\mu B)=0
$$

Equation (18) has six roots $\mu$, which are readily obtained by virtue of the fact that the equation can be analytically factored. The roots are zero (twice), $\left(u_{1} \pm c_{1}\right)^{-1}$, and $\left(u_{2} \pm\right.$ $\left.c_{2}\right)^{-1}$. The preceding analysis applies, of course, only if $\sigma \neq 0$. Thus, the sigma equation system, with $\sigma \neq 0$; has real characteristics.

The basic equation system $(\sigma=0)$ requires a separate characteristic analysis. This analysis can be carried out analy tically only for the incompressible case $\left(\rho_{1}=\right.$ constant, $\rho_{2}=$ constant): the compressible case must be solved numerically. In both cases, the characteristics are complex [1]. Thus, the introduction of surface tension into the basic equation system has the effect of changing the characteristics from complex to real. 


\section{LINEAR STABILITY ANALYSIS OF THE SIGMA EQUATION SYSTEM}

A linear stability analysis of the sigma equation system can be performed analy tically for the case of incompressible flow ( $\rho_{1}=$ constant, $\rho_{2}=$ constant) and an unperturbed solution having space and time derivatives of zero (that is, a uniform steady state). For incompressible flow, the equation system becomes

$$
\begin{gathered}
\frac{\partial \alpha_{i}}{\partial t}+u_{i} \frac{\partial \alpha_{i}}{\partial x}+\alpha_{i} \frac{\partial u_{i}}{\partial x}=0 \quad(i=1,2) \\
\frac{\partial u_{i}}{\partial t}+u_{i} \frac{\partial u_{i}}{\partial x}+\frac{\partial \hat{p}_{i}}{\partial x}=u \quad(i=1,2) \\
\alpha_{1}+\alpha_{2}=1 \\
\rho_{1} \hat{p}_{1}-\rho_{2} \hat{p}_{2}=-\sigma \frac{\partial^{2} \alpha_{1}}{\partial x^{2}}
\end{gathered}
$$

where $\hat{\mathrm{p}}_{\mathrm{i}}=\mathrm{p}_{\mathrm{i}} / \rho_{\mathrm{i}}$. To perform the stability analysis, each dependent variable $\mathrm{v}$ is replaced by $v+v^{\prime} \exp [i(k x-\omega t)]$, and the resulting equations are linearized with respect to the primed quantities. The assumption of a uniform steady state unperturbed solution means that $\mathrm{v}$ is independent of $x$ and $t$. The result of this replacement is

$$
\begin{aligned}
& \left(-i \omega+i k u_{1}\right) \alpha_{1}^{\prime}+i k \alpha_{1} u_{1}^{\prime}=0 \\
& -\left(-i \omega+i k u_{2}\right) \alpha_{1}^{\prime}+i k \alpha_{2} u_{2}^{\prime}=0 \\
& \left(-i \omega+i k u_{1}\right) u_{1}^{\prime}+i k \hat{p}_{1}^{\prime}=0 \\
& \left(-i \omega+i k u_{2}\right) u_{2}^{\prime}+i k \hat{p}_{2}^{\prime}=0 \\
& -\mathrm{k}^{2} \sigma H \alpha_{1}^{\prime}+\rho_{1} \hat{\mathrm{p}}_{1}^{\prime}-\rho_{2} \hat{\mathrm{p}}_{2}^{\prime}=0 .
\end{aligned}
$$

Equations (23) to (27) are a linear homogeneous system in the variables $\hat{p}_{1}^{\prime}, \hat{p}_{2}^{\prime}, u_{1}^{\prime}, u_{2}^{\prime}$, and $a_{1}^{\prime}$. For a nontrivial solution to exist, the determinant of coefficients must vanish. This condition leads to the equation 


$$
\alpha_{1} \rho_{2} \cdot\left(k u_{2}-\omega\right)^{2}+\alpha_{2} \rho_{1}\left(k_{1}-\omega\right)^{2}-\alpha_{1} \alpha_{2} \sigma \mathrm{H} k^{4}=0
$$

which determines the dispersion relations $\omega(\mathrm{k})$. The solutions of Equation (28) are

$$
\begin{aligned}
\omega & =\frac{k \alpha_{1} \alpha_{2}}{\alpha_{2} \rho_{1}+\alpha_{1} \rho_{2}}\left(\frac{\rho_{1} u_{1}}{\alpha_{1}}+\frac{\rho_{2} u_{2}}{\alpha_{2}}\right. \\
& \pm \sqrt{\left.\frac{\sigma \mathrm{H} k^{2}}{u_{1} u_{2}}\left(\alpha_{2} \rho_{1}+\alpha_{1} \rho_{2}\right)-\frac{\rho_{1} \rho_{2}}{u_{1} u_{2}}\left(u_{1}-u_{2}\right)^{2}\right)}
\end{aligned}
$$

The criterion for stability is $\operatorname{Im}(\omega) \leqslant 0$ for all roots $\omega$. In the present case, complex roots occur in conjugate pairs, so that $\omega$ must be purely real for stability. Equation (29) shows that instability results if the quantity under the radical is negative, whereas stability results if it is positive or zero. The condition for stability is therefore:

$$
\sigma \mathrm{Hk}^{2}\left(\alpha_{2} \rho_{1}+\alpha_{1} \rho_{2}\right) \geq \rho_{1} \rho_{2}\left(u_{1}-u_{2}\right)^{2}
$$

Unlike the characteristic analysis of the preceding section, the stability analysis is valid for an arbitrary value of $\sigma=0$. As a consequence, this analysis yields valuable information about both the sigma equation system and the basic equation system.

The trivial case $u_{1}=u_{2}$ is disposed of at the outset. In this case, stability is obtained for all wavelengths and all values of $\sigma \geqslant 0$. Henceforth, $u_{1}$ is assumed unequal to $u_{2}$.

By setting $\sigma=0$ in Equation (30), all wavelengths are seen to be unstable in the basic equation system. For the sigma equation system $\sigma$ is greater than zero, and Equation (30) shows that very short wavelengths (large $\mathrm{k}$ ) are stable, whereas very long wavelengths (small k) are unstable. For convenience, a critical wave number $\mathrm{k}_{\mathrm{c}}$ is defined by the equality sign in Equation (30):

$$
k_{c}=\left|u_{1}-u_{2}\right|\left(\frac{\rho_{1} \rho_{2}}{\sigma H\left(\alpha_{2} \rho_{1}+\alpha_{1} \rho_{2}\right)}\right)^{1 / 2} .
$$

Wavelengths longer than $2 \pi / \mathrm{k}_{\mathrm{c}}$ are unstable, whereas wavelengths shorter than or equal to $2 \pi / \mathrm{k}_{\mathrm{c}}$ are stable. As will be verified in the next section, the instability at long wavelengths is simply the well known Helmholtz instability.

Inspection of Equation (29) shows explicitly that the basic equation system and the sigma equation system exhibit virtually identical behavior when $k \ll k_{c}$; for in this case, the first term under the radical becomes negligible compared to the second and may be omitted, 
just as though $\sigma$ were zero. Therefore, for wavelengths long compared to $2 \pi / \mathrm{k}_{\mathrm{c}}$, the physical Helmholtz instability may be correctly calculated with either equation system. Thus, an equation system having complex characteristics can correctly predict long-wavelength physical instabilities.

At shorter wavelengths, however, the dispersion relation given by Equation (29), for a given value of $\sigma>0$, begins to differ appreciably from that for $\sigma=0$. Eventually, at wavelengths shorter than $2 \pi / \mathrm{k}_{\mathrm{c}}$, the two equation systems predict totally different behavior, the sigma system predicting stability and the basic system predicting instability. Now the sigma equation system is clearly physically the more realistic because surface tension is a real physical effect which should be included in a complete physical description. Therefore, the inference that the basic equation system exhibits incorrect and unphysical behavior at short wavelengths is justified (in particular, unphysical instability for $k>k_{c}$ ). This unphysical behavior, like the complex characteristics, results from the unwarranted neglect of surface tenision (or other short-wavelength effects, such as viscosity).

The growth factor corresponding to Equation (29) is

$$
G(k, t) \equiv\left|e^{-i \omega(k) t}\right|=e^{\operatorname{Im}(\omega) t} .
$$

When $\omega$ is complex, the positive sign in Equation (29) is the sign which corresponds to growth. By setting $\sigma=0$ in Equation (29):

$$
\operatorname{Im}(\omega)=\frac{k\left|u_{1}-u_{2}\right|}{\alpha_{2} \rho_{1}+\alpha_{1} \rho_{2}} \sqrt{\alpha_{1} \rho_{1} \alpha_{2} \rho_{2}}
$$

for the basic equation system. For the sigma equation system $\sigma>0$, and the corresponding result is

$$
\operatorname{Im}(\omega)=\left\{\begin{array}{l}
0 \text { if } k \geq k_{c} \\
k \sqrt{\frac{\alpha_{1} \alpha_{2} \sigma H}{\alpha_{2} \rho_{1}+\alpha_{1} \rho_{2}}\left(k_{c}^{2}-k^{2}\right)} \text { if } k<k_{c} .
\end{array}\right.
$$

Now the essence of a well posed problem is that, for a given time $t, G(k, t)$ be bounded with respect to the wavenumber $\mathrm{k}$ in the interval $0 \leqslant \mathrm{k}<\infty[8]$, which in turn requires that $\operatorname{Im}(\omega)$ be bounded with respect to $k$, which is true for Equation (34) but not for Equation (33). Thus, the basic equation system is shown to represent an improperly posed problem, whereas the sigma equation system represents a well posed problem. The fact that the basic equation system constitutes an improperly posed problem may indeed be regarded as due to a physical deficiency in the system - the failure to account for surface tension (or other short-wavelength effects). 


\section{EXACT TWO-DIMENSIONAL STABILITY ANALYSIS}

The conventional equations of multidimensional fluid dynamics are used in this section to analyze the behavior of separated two-phase flow between parallel plates including surface tension. The physical validity of the basic equations is well established, so that the results of the analysis can confidently be regarded as correct and can, therefore, be used to check the predictions of the one-dimensional, two-phase equations.

As in the preceding section, the tluids are assumed incomprcssible, and the motion of small perturbations about a uniform steady state solution is examined. The necessary mathematical development differs only slightly from that in Article 232 of Lamb $^{[9]}$ and hence will not be given in detail. Instead, only the modifications to Lamb's development which are necessary to treat the case of present interest are indicated.

The pressure interface condition is modified by adding the term $\sigma \partial^{2} \eta / \partial x^{2}$ to the left-hand side of Lamb's Equation (4) to allow for the presence of surface tension. The second modification is to allow for the finite depth of the two fluids by replacing the factors $e^{k y}$ and $e^{-k y}$ in Lamb's Equation (5) by the factors cosh $\left[k\left(y+\alpha_{1} H\right)\right]$ and cosh $\left[\mathrm{k}\left(\mathrm{y}-\alpha_{2} \mathrm{H}\right)\right]$, respectively. The third modification is to set $g=0$. The equation which then results in place of Lamb's Equation (9) is (in the notation of the present report):

$$
\begin{gathered}
\alpha_{1} \rho_{2}\left[\alpha_{2} \mathrm{kH} \operatorname{coth}\left(\alpha_{2} \mathrm{kH}\right)\right]\left(\mathrm{ku}_{2}-\omega\right)^{2} \\
+\alpha_{2} \rho_{1}\left[\alpha_{1} \mathrm{kH} \operatorname{coth}\left(\alpha_{1} \mathrm{kH}\right)\right]\left(\mathrm{ku}_{1}-\omega\right)^{2} \\
-\alpha_{1} \alpha_{2} \sigma \mathrm{H} \mathrm{k} \mathrm{k}^{4}=0 .
\end{gathered}
$$

Equation (35) determines the exact dispersion relations $\omega(\mathrm{k})$ for the physical problem under consideration. The validity of the sigma equation system may now be investigated by comparing Equation (28) with Equation (3b). Since $x$ coth $x \cong 1$ for $U<x<<1$, Equation (35) reduces to Equation (28) in the limit $\mathrm{kH}<<1$ (that is, when the wavelength is long compared to the spacing). In this limit, therefore, the sigma equation system exhibits correct physical behavior, at least with regard to small perturbations about a uniform steady state solution. When $\sigma$ is set equal to zero in Equations (28) and (35), the basic equation system is seen to exhibit correct physical behavior in the hypothetical case when the surface tension is identically zero. In this latter case, the problem is well known to be improperly posed, and the basic equation system appropriately reflects this behavior. In the case $\sigma>0$, the problem is well posed [as can explicitly be verified by solving Equation (35) for $\operatorname{Im}(\omega)$ ], and the sigma equation system reflects this behavior, even though it becomes quantitatively incorrect at short wavelengths. 
The failure of the sigma equation system at wavelengths comparable to the plate spacing was anticipated in the remark following Equation (8). It occurs because at such wavelengths the flow becomes essentially two dimensional in character and can no longer be described by a one-dimensional equation system.

The fact that the sigma equation system exhibits correct physical behavior when $\mathrm{kH} \ll<1$ places severe restrictions on the form of any further modifications to the system. In particular, any additional "interface force" terms added to the momentum equations must be such as to yield a vanishing contribution, except possibly at short wavelengths, in the problem of small perturbations about a uniform steady state solution.

However, a possible exception to this restriction should be mentioned. Such a restriction would clearly not apply if the objective were to obtain an equation system which does not attempt to describe small-amplitude disturbances correctly, but seeks instead to describe the average behavior of a "fully developed" two-phase flow in which apparently random motions reminiscent of turbulence appear to be superimposed on a slowly varying deterministic mean motion. This objective is indeed worthy but also uncommonly difficult, probably as difficult as that of constructing a complete theory of turbulence directly from the Navier-Stokes equations. In the present report, attention has been implicitly restricted to the problem of obtaining a satisfactory system of equations to describe the instantaneous motions in separated two-phase flow. 


\section{NUMERICAL CONSIDERATIONS}

Use of the sigma equation system to calculate a long-wavelength instability by a finite-difference numerical procedure is considered. If the wavelength of interest is long compared with $2 \pi / \mathrm{k}_{\mathrm{c}}$, whether the sigma equation system or the basic equation system is used is immaterial because both exhibit the same behavior for $\mathrm{k}<<\mathrm{k}_{\mathrm{c}}$. However, this statement implicitly assumes that care is taken not to introduce perturbations at other wavelengths having growth rates larger than that of the wavelength of interest. As Richtmyer and Morton .. [8] point out, the finite difference representation itself effectively introduces perturbations with wavelengths of the order of the mesh spacing $\Delta x$. Thus, instabilities at wavelengths of order $\Delta x$ can not be tolerated in a finite-difference calculation. This operational numerical constraint must be observed regardless of the equation system being used. Since all wavelengths, including those of order $\Delta x$, are unstable for the basic equation system, we would not expect to be able to successfully calculate even a long-wavelength instability using this system without introducing some artificial stabilizing effect at wavelength of order $\Delta x$.

In the sigma equation system, wavelengths of order $\Delta x$ may or may not be unstable, depending upon the magnitudes of $\Delta x$ and $\sigma$ and the values of the flow variables [Equation (30)]. In practice, for typical values of $\Delta x$ and the flow variables and typical physical surface tensions, wavelengths of order $\Delta x$ are still unstable; the stability condition [Equation (30)] is not satisfied until wavelengths considerably shorter than $\Delta \mathrm{x}$ are reached. The ideal solution to this problem would be to reduce $\Delta x$ until it becomes comparable to the (very small) value $2 \pi / \mathrm{k}_{\mathrm{c}}$. Unfortunately, reducing $\Delta \mathrm{x}$ to this extent will not ordinarily be economically feasible. Thus, even when the sigma equation system is used, introduction of an artificial stabilizing effect for wavelengths of order $\Delta \mathrm{x}$ will ordinarily be necessary.

The most straightforward way of introducing an artificial stabilizing effect would be to define an artificial surface tension $\sigma^{*}$, as suggested by Teller (quoted by Richtmyer and Morton ${ }^{[8]}$ ). The magnitude of $\sigma^{*}$ would be chosen in accordance with Equation (30) to be just sufficient to stabilize wavelengths of order $\Delta x$. By setting $k \cong \pi / \Delta x$ (the smallest wavelength which can be represented in the mesh is $2 \Delta x$ ), the following formula for $\sigma^{*}$ from Equation (30) is obtained:

$$
\sigma^{*}=c \frac{(\Delta x)^{2}}{\pi^{2} H}\left(\frac{\rho_{1} \rho_{2}}{\alpha_{2} \rho_{1}+\alpha_{1} \rho_{2}}\right)\left(u_{1}-u_{2}\right)^{2}
$$

where C is a dimensionless constant "safety factor" which should probably be set slightly larger than unity. As implied, $\sigma^{*}$ is typically much larger than physical values of the surface tension. Thus, even if initially the sigma equation system is used, the true surface tension can be neglected in comparison with the artificial one.

The preceding considerations suggest that the numerical calculations be performed using the sigma equation system with $\sigma$ replaced with $\sigma^{*}$, regardless of whether the sigma 
equation system or the basic equation system was originally advocated. From this point of view, which system was originally preferred does not seem to matter. However, from a more fundamental point of view, the system originally preferred does matter. If use of the basic equation system having complex characteristics had been advocated, we would effectively be switching to a different equation system having real characteristics at the point when $\sigma^{*}$ was introduced, regardless of the fact that it is introduced for a purely numerical reason.

Of course the use of $\sigma^{*}$ is not a unique way to achieve the desired stabilization of wavelengths of order $\Delta x$. Other artificial terms based on physical effects (for example, artificial viscosity or mass diffusion) lead to similar results. In addition, certain methods of differencing (in particular, "donor cells" or "upwind" differencing) themselves are well known to introduce an artificial stabilizing effect ${ }^{[10,11]}$, even though no additional stabilizing term has explicitly been put in. If such a differencing method is used, one may indeed be able to perform calculations in which the governing equations, which have complex characteristics, are apparently those of the basic equation system. However, an analysis of the differencing truncation errors would reveal the effective presence of a stabilizing term which, if it were included in the characteristic and stability analyses, would render the characteristics real and stabilize short-wavelength disturbances.

Finally, note should be taken of the fact that in particular special cases, calculations may be performed using the basic equation system with no artificial stabilizing effects of any kind, provided the entire calculation is finished before appreciable error growth has a chance to occur. This approach is clearly unsuitable for general use because it will work for calculations over "short" intervals in problem time, where the precise meaning of "short" is not known a priori. 


\section{CONCLUDING REMARKS}

In seeking to clarify the interrelationships of characteristics, stability, and shortwavelength phenomena in two-phase flow, consideration has been limited to a detailed analysis of the effect of surface tension. This particular short-wavelength effect was chosen primarily for convenience in the theoretical development. To physically include surface tension without also including viscosity is probably unreasonable, although to include viscosity and neglect surface tension might be reasonable. However, if an attempt had been made to analyze the effect of viscosity, an exact two-dimensional solution would not have been attained. Although the analysis of Sections II and III would not have been hindered and would have led to very similar results, the exact two-dimensional results of Section IV could not have been obtained because of the inapplicability of potential flow theory to viscous fluids. (In addition, the presence of viscosity would cause the velocity to vary much more over a cross-sectional plane perpendicular to the flow direction, which would make the variable-area equations on which our basic equations were based somewhat less satisfactory.)

Nevertheless, for the purpose of obtaining a two-phase flow equation system suitable for actual numerical calculations, reason exists to believe that viscosity is a more appropriate effect to include, than is surface tension, because viscosity is a dissipative effect, whereas surface tension is not ${ }^{[12]}$. Although surface tension exerts a stabilizing effect, it exerts no damping, and intuitively damping seems necessary for the equation system to be stable in some nonlinear sense. When a small perturbation of a given wavelength is introduced, the perturbation grows in accordance with the linear stability theory until its amplitude becomes so large that nonlinear terms become important. These nonlinear terms can be viewed as having the effect of transferring energy from the original wavelength into other wavelengths. Eventually, the entire wavelength spectrum becomes excited, and energy finds its way into the small stable wavelengths. In the case of surface tension, these wavelengths are stable but they are not damped. Their amplitude will, therefore, grow (not because of instability but because energy is being transferred into them from other wavelengths). When their amplitudes become large enough, the nonlinear terms will again come into play and will begin to transfer energy back out of the stable short wavelengths into the unstable longer wavelengths. Thus, in the nonlinear regime, all wavelengths would be expected to continue growing indefinitely, which is physically unacceptable because real physical systems are dissipative. Thus, the sigma equation system, although unquestionably more physical than the basic system, appears likely to exhibit unphysical behavior in the nonlinear regime.

In the case of viscosity, however, the short wavelengths are actually damped so that once kinetic energy is transferred into them, it is dissipated as heat and effectively removed from the system. Thus, a sink exists in the spectrum at short wavelengths whereby kinetic energy can leave the system. A situation can be pictured, reminiscent of turbulence in which energy is continually being extracted from the mean motion, transferred in a cascade process to shorter and shorter wavelengths and ultimately dissipated in the very short wavelengths to heat. No wavelength in the spectrum will then grow indefinitely; its amplitude will reach an equilibrium in which the rate at which it acquires energy directly, as a result of its instability, is equal to the net rate at which it transfers energy to other wavelengths. 
Although speculative in nature, the preceding remarks suggest that the primary usefulness of the sigma equation system is more likely to be theoretical, as in the present report, than practical. One suspects $a$ priori that the equation system obtained by incorporating viscosity into the basic equation system would give a better representation of physical reality in the nonlinear regime and hence be more suitable for practical applications.

The preceding qualitative remarks also suggest that methods and concepts from turbulence theory may prove useful in formulating a theory of the average behavior of "fully developed" two-phase flow, as discussed at the end of Section IV. The authors are currently considering this possibility.

Finally, we remark that much of the concern over complex characteristics in two-phase flow appears to have been due to the fact that characteristics and stability, although not unrelated as shown in Appendix A, are in reality two separate concepts which should not be confused. Indeed, in a practical sense, characteristics are largely irrelevant; it is stability. about which one should be most concerned when analyzing the behavior of equation systems. As discussed at the end of Section. III, it is stability rather than characteristics which is directly related to the question of well posedness. Theorems relating characteristics to well posedness typically restrict attention to the case of constant coefficients (for example, Lax ${ }^{[13]}$ ), in which stability and characteristics are trivially equivalent as shown in Appendix A. If attention had been focused at the outset on stability rather than characteristics in two-phase flow; realization might have come more quickly that the single most essential modification to the basic equation system is the introduction of short-wavelength effects such as surface tension or viscosity. In retrospect, this modification is clearly suggested by the well known fact that such effects are sufficient to stabilize short wavelengths and produce properly posed problems in other contexts ${ }^{[.8]}$. 


\section{REFERENCES}

1. D. Gidaspow (chairman), "Modeling of Two-Phase Flow", proceedings of Round Table Discussion RT-1-2 at the Fifth International Heat Transfer Conference, Tokyo, Japan, September 3-7,1974, also in Heat Trans., 3 (1974).

2. H.W. Liepmann and A. Roshko, Elements of Gasdynamics, New York: John Wiley and Sons, 1957, pp 40, 48.

3. P.R. Garabedian, Martial Differential Equations, New York: John Wiley and Sons, 1964.

4. R. Courant and D. Hilbert, Methods of Mathematical Physics, Vol. II, New York: Interscience, 1962.

5. J. Hadamard, Lectures on Cauchy's Problem in Linear Partial Differential Equations, New Haven: Yale University Press, 1923.

6. S. I. Pai, "A critical Review of the Fundamental Equations of a Mixture of a Gas and Small Solid Particles", Z. Flugwiss., 19 (1971) pp 353-360.

7. J. Boure et al, "Choking Flows and Propagation of Small Disturbances", European Two-Phase Flow Group Meeting, Brussels, Belgium, 1973, Paper F1.

8. R. D. Richtmyer and K. W. Morton, Difference Methods for Initial-Value Problems, 2nd ed, New York: Interscience, 1967, pp 351-360.

9. H. Lamb, Hydrodynamics, 6th ed, New York· Dover Puhlications, 1945.

10. F. H. Harlow and A. A. Amsden, "A Numerical Fluid Dynamics Calculation Mcthod for All Flow Speeds", J. Comp. Phys., 8 (1971) pp 197-213.

11. K. W. Morton, "Stability and Convergence in Fluid Flow Problems", Proc. Roy. Soc. Lond. A., 323 (1971) pp 237-253.

12. J. M. Delhaye, "Jump Conditions and Entropy Sources in Two-Phase Systems. Local Instant Formulation", Int. J. Multiphase Flow, 1, (1974) pp 395-409.

13. P.D. Lax, "Differential Equations, Difference Equations, and Matrix Theory", Comm. Pure Appl. Math., 11 (1958) pp 175-194. 
APPENDIX A

RELATIONSHIP BETWEEN CHARACTERISTICS AND STABILITY

$\therefore$ 
THIS PAGE

\section{WAS INTENTIONALLY LEFT BLANK}




\section{APPENDIX A}

\section{RELATIONSHIP BETWEEN CHARACTERISTICS AND STABILITY}

In this appendix, the relation between characteristics and stability for a system of $n$ first-order, quasi-linear partial differential equations of the form

$$
A(v) \cdot \frac{\partial v}{\partial t}+B(\underset{\sim}{v}) \cdot \frac{\partial v}{\partial x}+c(v)=0
$$

is clarified. In Equation (A-1), $\underset{\sim}{\mathrm{v}}$ is a $n$-dimensional column vector of dependent variables, $\mathbf{A}$ and $\mathrm{B}$ are $n \times n$. square matrices, and $\underset{\sim}{\mathrm{C}}$ is a $n$-dimensional source vector. The elements of $\mathbf{A}$, $B$, and $\underset{\sim}{c}$ are unspecified functions of the components of $\underset{\sim}{v}$

The characteristic roots $\mu$ of the system [Equation (A-1)] (which for brevity are referred to as characteristics throughout the report) are defined by the equation

$$
\operatorname{det}\left(A-\mu_{B}\right)=0 \text {. }
$$

(The source vector $\underset{\sim}{\mathrm{c}}$ is by definition not included in the characteristic analysis:)

The characteristics $\mu$ defined by Equation (A-2) are the reciprocals of those customarily used $[A-1,-2]$. The reason for this choice is that if one of the matrices is singular, it will usually be $\mathbf{A}$ rather than $\mathbf{B}$. If $\mathbf{A}$ is singular and $\mathbf{B}$ is nonsingular, Equation (A-2) still possesses $n$ roots. Some of these roots may be zero. If so, the corresponding reciprocal roots $\lambda=1 / \mu$ are infinite and would not be obtained by solving the equation $\operatorname{det}(\lambda \mathbf{A}-\mathbf{B})=0$.

Next, the local linear stability behavior of Equation $(A-1)$ is examined. To do so, $\mathfrak{v}$ is replaced by $\underset{\sim}{\mathrm{v}}+\delta \underset{\sim}{\mathrm{v}}$, and the result is linearized with respect to $\delta \underset{\sim}{\mathrm{v}}$, resulting in

$$
\begin{aligned}
& A(\underline{v}): \frac{\partial}{\partial t}(\delta \underline{v})+B(\underline{v}) \cdot \frac{\partial}{\partial x}(\underset{\sim}{v})+\left(\underline{\sim} \cdot \frac{\partial A}{\partial \underline{v}}\right): \frac{\partial \underline{v}}{\partial t} \\
& +\left(\delta \underline{v} \cdot \frac{\partial \mathrm{B}}{\partial \underline{\sim}}\right) \cdot \frac{\partial \underset{\sim}{v}}{\partial \mathrm{x}}+\dot{\delta v}: \frac{\partial \underline{\sim}}{\partial \underline{\sim}}=0 .
\end{aligned}
$$

Equation (A-3) describes small perturbations $\delta \underset{\sim}{v}$ about an unperturbed solution with instantaneous values of $v, \partial v / \partial t$, and $\partial v / \partial x$. These instantaneous values are treated as constants and a solution of the form

$$
\delta \underline{\sim}=\delta \underline{\sim}_{0} \exp [i(k x-\omega t)]
$$


is assumed. Equation (A-3) then becomes

$$
\begin{aligned}
& -i \omega A(\underline{v}) \cdot \delta \underline{\sim}_{0}+i k B(\underline{v}) \cdot \delta \underline{\sim}_{0}+\left(\delta \underline{\sim}_{0} \cdot \frac{\partial A}{\partial \underline{v}}\right) \cdot \frac{\partial \underline{v}}{\partial t} \\
& +\left(\delta{\underset{\sim}{\mathrm{o}}}_{0} \cdot \frac{\partial \mathrm{B}}{\partial \mathrm{v}}\right): \frac{\partial \mathrm{v}}{\partial \mathrm{x}}+\delta \underline{\sim}_{0} \cdot \frac{\partial \underset{\sim}{\sim}}{\partial \underset{\sim}{v}}=\cdot 0
\end{aligned}
$$

Equation (A-5) is a homogeneous linear system in the components of $\delta \mathrm{v}_{0}$. In order for a nontrivial solution to exist, the determinant of the coefficient matrix must vanish. The dispersion relations $\omega(\mathrm{k})$ are, therefore, determincd by

$$
\operatorname{det}(-i \omega A+i k B+D)=0
$$

where

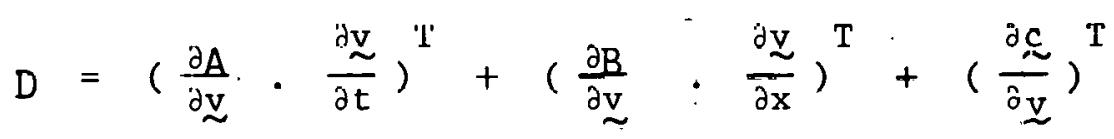

with the superscript $\mathrm{T}$ denoting the matrix transpose. If $\omega(\mathrm{k})$ is assumed nonzero, Equation (A-6) may be rewritten as

$$
\operatorname{det}\left(A-\frac{k}{\omega} B+\frac{i}{\omega} D\right)=0
$$

Because of the presence of $i$ in Equation (A-8), the complex roots $\omega(k)$ corresponding to a given real value of $k$ need not occur in conjugate pairs. Inspection of Equation (A-4) shows that the condition for stability is that $\operatorname{Im}(\omega)$ be $\leqslant 0$ for all roots $\omega$.

Comparison of Equation (A-2) and (A-8) shows that the characteristics and stability questions differ because of the presence of $D$ in Equation ( $A$ 8). If $D$ were zero, the dispersion relations could be obtained from the characteristics simply by equating $(\mathrm{k} / \omega)$ to $\mu$. Equation (A-7) shows that $\mathbf{D}$ equals zero trivially when $\mathbf{A}, \mathbf{B}$. and $\underset{\sim}{\mathrm{f}}$ are constants independent of $\underset{\sim}{\mathrm{v}}$. When $\mathbf{A}$ or $\mathbf{B}$ depend on $\underset{\sim}{\mathrm{v}}$, a nonzero $\mathbf{D}$ will result when the unperturbed solution is not constant in time or space. A nonzero $\mathbf{D}$ will also result if the source vector $\underset{\sim}{\mathrm{c}}$ is nonzero and depends upon $\underset{\sim}{\sim}$

Thus, if the equation system has real characteristics and no source terms, then a uniform steady state solution is necessarily stable with respect to small disturbances. This stability is neutral - small disturbances neither grow nor decay with time. However, when D $\neq 0$, real characteristics are not sufficient to ensure stability; this fact explains how systems with real characterictics can exhibit physical instabilities and clearly indicates that complex characteristics are not necessary to describe such instabilities. 
Although stability and characteristics are not related in general, they become essentially equivalent (with an exception to be noted) in the limit of very high frequencies (large $\omega$ ). This equivalence can be seen intuitively by inspection of Equation (A-8), where D is seen to be multiplied by $1 / \omega$. This intuitive expectation may be made rigorous by means of a theorem due to Ostrowski $[\mathrm{A}-3]$ : If the assumption is made that $\mathrm{k} /|\omega(\mathrm{k})|$ approaches a finite limit as $\mathrm{k} \rightarrow \infty$, as is invariably the case, Ostrowski's theorem then implies that

$$
\lim _{k \rightarrow \infty}\left|\mu-\frac{k}{\omega}\right|=0
$$

where $\mu$ is the characteristic root corresponding to the stability root $k / \omega$. Equation (A-9) shows that the characteristic and stability roots become arbitrarily close together in the limit of large k. First, the case for which $\mu$ is real is considered. Equation (A-9) then implies that as $\mathrm{k} \rightarrow \infty$, the imaginary part of $\mathrm{k} / \omega$ approaches zero, whereas the real part approaches $\mu$. Thus, provided $\mu \neq 0$, the imaginary part of $\omega$ becomes small compared to the real part as $k \rightarrow \infty$, so that if unstable growth occurs at all, it occurs at worst on a time scale much slower (in the limit, infinitely slower) than the period of the harmonic oscillations. In this sense, the motion may be regarded as almost stable; thus, if all the characteristic roots $\mu$ are real and nonzero, then the motion is either stable or almost stable at very short wavelengths.

However, if $\mu=0$ for one or more roots this conclusion does not follow, and no information is available about the growth rate of any residual instabilities which may remain as $\mathrm{k} \rightarrow \infty$, regardless of the fact that all the $\mu$ are real. The case $\mu=0$ is thus the exception to the relation between characteristics and stability which was referred to previously. As seen in Section II, the sigma equation system is of this type. One might be tempted too conjecture, on the basis of the results of Section III, that stability at very short wavelengths results when all the $\mu$ are real even if some of them are zero. Such a conjecture cannot be correct without qualification, however; a counterexample is provided by considering $\sigma$ to be negative. If $\sigma<0$, the characteristic analysis of Section II is unaltered, but an unbounded instability is introduced as $k \rightarrow \infty$ [Equation (29) of the body of this report].

In the case for which $\mu$ is complex $\left(\mu=\mu_{\mathrm{R}}+\mathrm{i} \mu_{\mathrm{I}}, \mu_{\mathrm{I}}, \neq 0\right)$, Equation (A-9) implies that the imaginary part of $k / \omega$ approaches $\mu_{I}$ as $k \rightarrow \infty$; that is:

$$
\lim _{k \rightarrow \infty}\left[\frac{-\operatorname{Im}(\omega) k}{|\omega|^{2}}\right]=\mu_{I}
$$

But $\mathrm{k} / \mathrm{| \omega} \mid$ is assumed to approach a finite limit, which may be positive or zero, as $\mathrm{k} \rightarrow \infty$. Thus, $|\omega|^{2} / \mathrm{k} \rightarrow+\infty$ as $\mathrm{k} \rightarrow \infty$. Since the complex characteristics occur in conjugate pairs, $\mu_{\mathrm{I}}$ may be considered negative. Equation (A-10) then implies that $\operatorname{Im}(\omega) \rightarrow+\infty$ as $k \rightarrow \infty$. Thus, an instability results at very short wavelengths; moreover, the growth rate is unbounded as $\mathrm{k}: \rightarrow \infty$ so that the problem is improperly posed. 
The preceding analysis applies only when the derivatives $\underset{\sim}{\partial v} / \partial \mathrm{t}$ and $\underset{\sim}{\partial} / \partial \mathrm{x}$ of the unperturbed solution are finite; otherwise $D / \omega$ in Equation $(\widetilde{A}-8)$ can not be made arbitrarily small by increasing $\mathrm{k}$. Unbounded derivatives appear to be the reason the usual equations of inviscid incompressible single-phase fluid dynamics, whose characteristics are real, can predict instability as $\mathrm{k} \rightarrow \infty$ in the classical Helmholtz problem with zero surface tension: the density and velocity are discontinuous at the interface so that the unperturbed solution has infinite space derivatives.

The develupment piesented in this appendix has hecn based upon a type of stability analysis which we refer to as local linear stability analysis. The adjective "local" refers to the fact that the coefficients in the linearized Equation (A-3) were treated as constants in order to admit solutions of the form given in Equation (A-4). In a rigorous linear stability analysis, these coefficients would he treated as given functions of $x$ and $t$ determined by the unperturbed solution $\underset{\sim}{\mathrm{v}}(\mathrm{x}, \mathrm{t})$. The coefficients are then variable and $\underset{\sim}{\delta} \mathrm{v}$ must be solved for by numerical procedures. The local linear stability analysis is less rigorous but very useful, as has been shown, in obtaining a substantially correct understanding with a minimum of computational labor. This method of analysis appears first to have been used by Von Neumann and Richtmyer $[\mathrm{A}-4]$. The stability analyses performed in Sections III and IV, however, were rigorous linear stability analyses, which could be analytically performed because the coefficients were constant for the particular unperturbed solution under consideration.

\section{REFERENCES}

A-1. P. R. Garabedian, Partial Differential Equations, New York: John Wiley and Sons, 1964.

A-2. R. Courant and D. Hilbert, Method of Mathematical Physics, Vol. II, New York: Interscience, 1962.

A-3. J. H. Wilkinson, the Algebraic Eigenvalue Problem, London: Oxford University Press, $1965, \mathrm{p} 63$.

A-4. J. Von Neumann and R. D. Richtmyer, "A Method for the Numerical Calculation of Hydrodynamic Shocks", J. Appl. Phys., 21 (1950) pp 232-237. 


\section{DISTRIBUTION RECORD FOR ANCR-1272}

\section{Externa]}

296 - NRC-4 - Water Reactor Safety Research Analys is Development

2 - H. J. C. Kouts, NRC

Internal

1. - Chicago Patent Group - ERDA 9800 South Cass Avenue

Argonne, Illinois 60439

3 - A. T. Morphew, Classification and Technical Information Officer ERDA-ID

Idaho Fall's, Idaho 83401

I - R. J. Beers, ID

1 - P. E. Litteneker, ID

1 - R. E. Swanson, ID

I - V. A. Walker, ID

1 - R. E. Wood, ID

11 - Special Internal Distribution

11 - INEL Technical Library

40 - Authors 